\title{
Section-Wise Similarities for Classification of Subjective-Data on Time Series
}

\author{
Isaac Martín de Diego, Oscar S. Siordia, Cristina Conde, \\ and Enrique Cabello \\ Face Recognition and Artificial Vision Group, \\ Universidad Rey Juan Carlos, \\ C. Tulipán, S/N, 28934, Móstoles, España \\ \{isaac.martin, oscar.siordia, cristina.conde, enrique.cabello\}@urjc.es
}

\begin{abstract}
The aim of this paper is to present a novelty methodology to develop similarity measures for classification of time series. First, a linear segmentation algorithm to obtain a section-wise representation of the series is presented. Then, two similarity measures are defined from the differences between the behavior of the series and the level of the series, respectively. The method is applied to subjective-data on time series generated through the evaluations of the driving risk from a group of traffic safety experts. These series are classified using the proposed similarities as kernels for the training of a Support Vector Machine. The results are compared with other classifiers using our similarities, their linear combination and the raw data. The proposed methodology has been successfully evaluated on several databases.
\end{abstract}

Keywords: Similarity, Kernel Method, Classification, Time Series, Data Segmentation.

\section{Introduction}

Similarity measures between time series is a common issue that has been treated in several ways. Usually, the statistical models fitted to the series are compared. Nevertheless, subjective-data time series are rarely taked into account. This kind of data corresponds to information collected from human opinions over a period of time. In this case, it is not possible to successfully fit a unique model to all the data set since the changes on the level of the series usually respond to a great variety of factors. In the particular case of driving risk evaluations from human experts, these factors are related to driver's responses to vehicle or road variations (speed, aceleration, road conditions, etc). The driver's distraction study is a very difficult problem due to the high number of factors involved in the distraction-related accidents 11. However, these distractions can be reduced with the development of a system that can automatically evaluate the driver's behavior. CABINTEC ("Intelligent cabin truck for road transport") is an ongoing project focused on risk reduction for traffic safety [2]. One of the main objectives of the project is to identify driver's unsuitable behavior and lacks of attention. For that purpose, a system for automatic driving risk evaluation is 
being developed using time series information from several traffic safety experts. Further, a previous selection of experts is needed in order to detect unpracticed experts whose opinions should not be considered. For this purpose, it is necessary to measure the similarity between experts risk evaluations time series.

Most of the known similarity measures between time series are concerned with the distance between series levels. However, for subjective data, it is necessary to define a measure that considers the similarity in the behavior (trend of data) as well as the similarity in the level. For this purpose, a proper representation of the time series is needed. The same idea for signal matching has a long history in cardiac signal analysis (see, for instance, [3]). However, most of these representations imply sensitivity to noise, lack of intuitiveness, and the need to fine-tune many parameters [4. In this paper, an alternative representation based on a linear segmentation of the time series is proposed. The segmented representation allows the definition of two similarity measures considering the behavior of the series and the series level, respectively. The defined similarities were used as kernels to train a Support Vector Machine (SVM) and a k-NN (kNearest Neighbor) classifier. The proposed methodology, main contribution of this paper, was applied for the classification of a group of traffic safety experts labeled according to their professional experience. The data acquisition process was made as follows: a driving simulation exercise of ten minutes was recorded from a truck cabin simulator from the Centro de Estudios e Investigaciones Técnicas de Gipuzkoa (CEIT) 2]. Then, a group of 47 traffic safety experts were asked to evaluate the driving risk of the simulated exercise. For that purpose, the simulation reproduction tool Virtual Co-driver was used [5]. To collect the experts evaluations, a Visual Analog Scale (VAS) was employed. This method has been considered the best for subjective measurements (see, for instance, [6]). The considered VAS ranges from 0 to 100, where 100 refers to the highest driving risk level. Finally, to know some aspects of experts experience in the traffic safety field, a personal quiz was applied.

The rest of the paper is organized as follows. Section 2 presents our algorithm for the linear segmentation of time series. In Section 3. the section-wise similarities are defined. Several experiments on real databases are considered in Section 4 to evaluate the relative performance of the proposed similarities. Section 5 concludes this paper.

\section{Linear Segmentation Algorithm}

In order to define a similarity between time series, small oscillations out of the main trend could be uninformative and generate noise. In this case, these oscillations should be removed. This happens, for instance, when dealing with subjective-data time series, where a linear behavior along a temporary period of time is expected. A variety of algorithms have been proposed to obtain proper representations of time series (see, for instance, [7] and [8]). In this paper, an algorithm to reduce the complexity of the data through the fit of regression lines in local sections of the series has been developed. The linear model was selected 


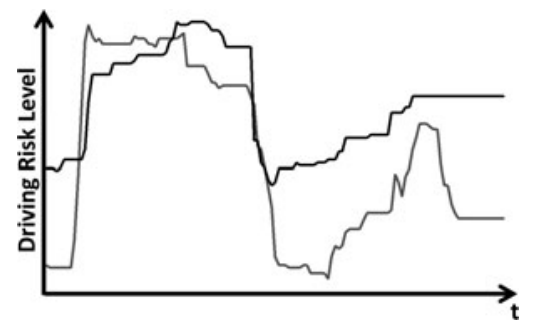

(a) Example of expert (red line) and unpracticed evaluators (black line).

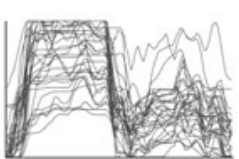

(b) Expert

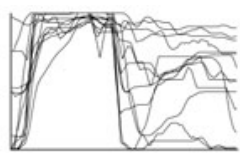

(c) Unpracticed

Fig. 1. Labeled CABINTEC time series in accordance with the traffic safety experience of the human evaluator: (a) example series of an expert and an unpracticed evaluator, (b) series of the experts evaluations, and (c) series of the unpracticed evaluators

due to its low computational cost. To illustrate the algorithm an example of the CABINTEC time series will be used (Figure 1(a) . The key idea of the algorithm is to represent the time series into a section-wise linear approximation losing the least amount of usefull information. The output will be a set of estimated regression lines, one per section. A scheme of the algorithm is presented in Algorithm 1.

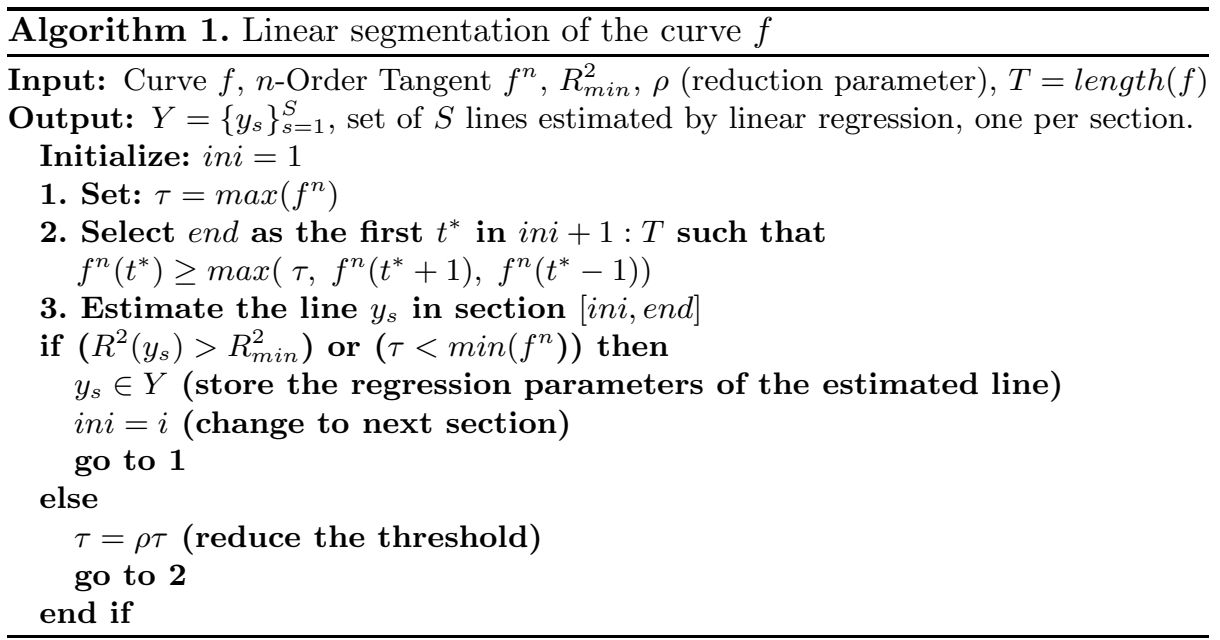

The start and end points of each section must denote a change on the trend of the serie. For that purpose the tangent of the time serie at each point needs to be defined. Let $f(t)$ be the value of curve $f$ evaluated in time $t=\{1, \ldots, T\}$. Let $f^{n}(t)$ the value of its tangent of order $n$, calculated as: 
Table 1. Linear Segmentation Algorithm on CABINTEC data

\begin{tabular}{|c|c|c|c|c|c||c|}
\hline$R_{\min }^{2}$ & 0.40 & 0.70 & 0.80 & 0.90 & 1.00 & $\begin{array}{c}\text { Original } \\
\text { Data }\end{array}$ \\
\hline $\begin{array}{c}\text { Number of } \\
\text { Sections }\end{array}$ & 2 & 10 & 18 & 23 & 130 & $\begin{array}{c}131 \\
\text { registers }\end{array}$ \\
\hline $\begin{array}{c}\text { Segmented } \\
\text { Graph Result }\end{array}$ & $n$ & $\Omega$ & $\Omega$ & & \\
\hline
\end{tabular}

$$
f^{n}(t)=h f(t)-\sum_{i=-n, i \neq 0}^{n} h_{i} f(t+i),
$$

where $h_{i}=1 /(2|i|)$, and $h$ is the sum of all $h_{i}$.

Notice that the weight $\left(h_{i}\right)$ assigned to the $i$-th neighbor of the point $t$ is inversely proportional to the distance between them.

The basis of this time series segmentation algorithm is to look for strong changes in the trend of data. This aim is achieved by the search of local maxima of $f^{n}$. When a point is selected in step 2 of Algorithm 1, a section $s$ in the serie is defined, and a regression line is fitted to the data in that section. Let $R_{y_{s}}^{2}$ be the square of the sample correlation coefficient of the regression line. If the fitted model explains much of the variability of the section, the calculated parameters of the regression line are stored and the algorithm iterates. In other case, no linear behavior is expected. Therefore, the section needs to be subdivided.

An example of the algorithm performance is presented in Table 11. The segmented graphical result and the number of sections generated by the algorithm were calculated for different selections of $R_{\text {min }}^{2}$, the minimum fit (or one minus the maximum error) allowed. For this example a tangent of order 5 was used. When $R_{\min }^{2}=0.40$, only two sections were generated. It is clear that the unique cut point was given by the global maximum of the 5-order tangent. Notice that the higher the $R_{\text {min }}^{2}$ was, the higher the number of sections were obtained. In the case of $R_{\text {min }}^{2}=1$, a section is generated for each pair of points. To choose the optimal linear representation of the database we consider a tradeoff between the global linear error and the complexity of the representation as follows:

$$
\alpha\left(1-R^{2}\right)+(1-\alpha) \frac{\text { number of sections }}{T-1} .
$$

In the example, $R_{\text {min }}^{2}=0.90$ minimizes expression (2) (trade-off parameter $\alpha=0.5$, which implies similar relevance for both terms).

\section{Section-Wise Similarities}

Given a set of segmented time series, it is possible to calculate several sectionwise similarities between them. Next, we propose one similarity measure based 


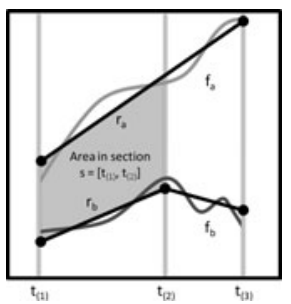

(a) Existent Area

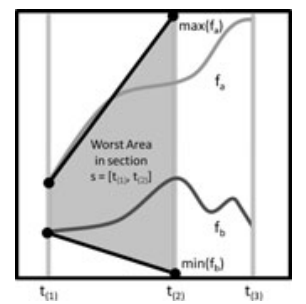

(b) Worst Area

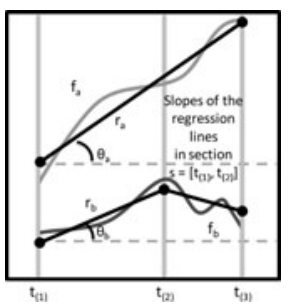

(c) Existent Angle

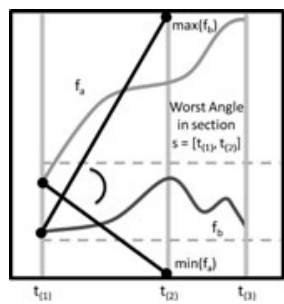

(d) Worst Angle

Fig. 2. Level and Angle based section-wise similarities of the time series $f_{a}$ and $f_{b}$, in the section between the points $t_{(1)}$ and $t_{(2)}$

on the differences between the level of the sections lines, and one similarity measure based on the differences between the angles of the sections lines.

Let $f_{a}$ and $f_{b}$ be two different time series. Let $\{A\}$ and $\{B\}$ be the set of initial and final points that defined the sections obtained for $f_{a}$ and $f_{b}$, respectively, from Algorithm[1. The similarity measures will be built on sections defined from the union of sets $\{A\}$ and $\{B\}$. That is, every initial or final point of a section from $f_{a}$ or $f_{b}$ will be used.

\subsection{Level Based Similarity}

Let $s=\left[t_{(1)}, t_{(2)}\right]$ be the section where $t_{(1)}$ and $t_{(2)} \in\{A\} \cup\{B\}$. Let $r_{a}$ and $r_{b}$ be the regression lines obtained from Algorithm 1 for $f_{a}$ and $f_{b}$, respectively, in $s$ (see Figure 2(a). Consider the area $L$ bounded by $r_{a}$ and $r_{b}$ calculated as:

$$
L=\frac{1}{2}\left[\left(r_{a}\left(t_{(1)}\right)-r_{b}\left(t_{(1)}\right)\right)+\left(r_{a}\left(t_{(2)}\right)-r_{b}\left(t_{(2)}\right)\right)\right] *\left(t_{(2)}-t_{(1)}\right),
$$

where $r_{i}\left(t_{(j)}\right)$ denotes the value of the regression line $r_{i}$ in the point $t_{(j)}$.

Notice that $\left(t_{(2)}-t_{(1)}\right)$ is the width (number of points) of section $s$. The level based similarity calculated in the section $s$, denoted by $s_{0 S}(s)$, is obtained as the relation between the area in (3) and the worst possible area $\breve{L}$ as follows: $s_{0 \mathrm{~S}}(s)=1-L / \breve{L}$. Notice that in this way, the proposed similarity measure is in $[0,1]$. The worst area $\breve{L}$ is calculated from the maximum possible change in the analyzed section. For that purpose, a line from $f_{b}\left(t_{(1)}\right)$ to $\min \left(f_{b}\right)$ and a line from $f_{a}\left(t_{(1)}\right)$ to $\max \left(f_{a}\right)$ are considered, as shown in Figure 2(b). Finally, the overall level based section-wise similarity for the time series $f_{a}$ and $f_{b}$ is calculated as the weighted sum of all the sectional similarities as follows:

$$
S_{0 \mathrm{~S}}\left(f_{a}, f_{b}\right)=\frac{\sum_{s=1}^{S} w(s) s_{0 \mathrm{~S}}(s)}{\sum_{s=1}^{S} w(s)},
$$

where $w(s)$ is the time width of section $s=1, \ldots, S$. 


\subsection{Angle Based similarity}

The angle based section-wise similarity considers the angle formed by the regression lines $r_{a}$ and $r_{b}$ defined in the section $s$. Let $\theta_{a}$ and $\theta_{b}$ be the slopes of $r_{a}$ and $r_{b}$, respectively (see Figure 2(c) . The angle between the regression lines is calculated as:

$$
\theta_{a b}=\left|\theta_{a}-\theta_{b}\right| \text {. }
$$

The angle based similarity calculated in the section $s$, denoted by $s_{1 \mathrm{~S}}(s)$, is obtained as the relation between the angle $\theta_{a b}$ and the worst possible angle $\breve{\theta}$ : $s_{1 \mathrm{~S}}(s)=1-\theta_{a b} / \breve{\theta}$. The worst angle $\breve{\theta}$ is calculated with the crossed maximum possible change in the analyzed section. That is, a line from $f_{b}\left(t_{(1)}\right)$ to $\max \left(f_{b}\right)$ and a line from $f_{a}\left(t_{(1)}\right)$ to $\min \left(f_{a}\right)$ as shown in Figure $2(\mathrm{~d})$ are considered. Finally, the overall angle based section-wise similarity for the time series $f_{a}$ and $f_{b}$ is calculated as the weighted sum of all the sectional similarities as follows:

$$
S_{1 \mathrm{~S}}\left(f_{a}, f_{b}\right)=\frac{\sum_{s=1}^{S} w(s) s_{1 \mathrm{~S}}(s)}{\sum_{s=1}^{S} w(s)},
$$

where $w(s)$ is the time width of section $s=1, \ldots, S$.

\section{Experiments}

The CABINTEC time series (Figure 1), were labeled in accordance with the experience in traffic safety of each human evaluator. Each evaluator was labeled as expert or unpracticed depending on their profession and years of experience in the field (Figure 1(b) and Figure 1(c)]. This information was obtained from personal quizzes applied to each evaluator before the knowledge acquisition experiments.

In order to test the accuracy and utility of similarities defined in Section 3 . several classification experiments were performed. These similarities were used as kernel to train a SVM following [9] $(C=100)$. Further, the similarity matrices were applied to a 1-Nearest Neighbor classifier. In addition, for benchmarking, two similarities based on the Euclidean distance were considered. Let $f_{a}$ and $f_{b}$ be two time series. $S_{0 \mathrm{P}}$ denotes a point-wise similarity based on the Euclidean

Table 2. Summary of the databases considered in the classification experiments

\begin{tabular}{|c|c|c|c|c|}
\hline Database Name & $\begin{array}{c}\text { Number of } \\
\text { Classes }\end{array}$ & $\begin{array}{c}\text { Number of } \\
\text { series }\end{array}$ & $\begin{array}{c}\text { Time Series } \\
\text { Length }\end{array}$ & Train set $\%$ \\
\hline \hline CABINTEC & 2 & 47 & 313 & 50 \\
\hline Gun Point & 2 & 200 & 150 & 25 \\
\hline ECG200 & 2 & 200 & 96 & 50 \\
\hline Coffee & 2 & 56 & 286 & 50 \\
\hline Growth & 2 & 93 & 31 & 64 \\
\hline
\end{tabular}


Table 3. Error rate and (standard deviation) of the classification experiments using section-wise similarities (single and combined), point-wise similarities (single and combined), SVM on raw data and NFDA on raw data

\begin{tabular}{|c|c|c|c|c|c|c|}
\hline $\begin{array}{c}\text { Classification } \\
\text { Algorithm } \\
\end{array}$ & $\begin{array}{c}\text { Similarity } \\
\text { Applied }\end{array}$ & CABINTEC & Gun Point & ECG200 & Coffee & Growth \\
\hline \multirow{6}{*}{ SVM } & $S_{\mathrm{OS}}$ & $18.8 \quad(0.21)$ & $5.0 \quad(0.07)$ & $12.6 \quad(0.10)$ & $19.6 \quad(0.25)$ & $5.8 \quad(0.11)$ \\
\hline & $S_{1 \mathrm{~S}}$ & $29.4 \quad(0.25)$ & $\begin{array}{ll}14.3(0.10)\end{array}$ & $20.6 \quad(0.11)$ & $2.9(0.09)$ & $6.0 \quad(0.10)$ \\
\hline & $\frac{S_{0 \mathrm{~S}}+S_{1 \mathrm{~S}}}{2}$ & $10.2(0.21)$ & $\begin{array}{ll}6.9 & (0.08) \\
\end{array}$ & $16.0 \quad(0.10)$ & $\begin{array}{ll}3.2 & (0.12) \\
\end{array}$ & $\begin{array}{ll}5.0 \quad(0.10) \\
\end{array}$ \\
\hline & $s_{0 P}$ & $\begin{array}{ll}19.5 \quad(0.20) \\
\end{array}$ & $\begin{array}{ll}6.8 \quad(0.10) \\
\end{array}$ & $11.8 \quad(0.10)$ & $\begin{array}{ll}8.8 & (0.22) \\
\end{array}$ & $\begin{array}{ll}4.9 \quad(0.10) \\
\end{array}$ \\
\hline & $S_{1 \mathrm{P}}$ & $32.4 \quad(0.23)$ & $\begin{array}{ll}6.7 & (0.11)\end{array}$ & $\begin{array}{ll}19.6 \quad(0.11) \\
\end{array}$ & $\begin{array}{ll}5.2 & (0.15) \\
\end{array}$ & $\begin{array}{ll}6.6 \quad(0.12) \\
\end{array}$ \\
\hline & $\frac{S_{0 \mathrm{P}}+S_{1 \mathrm{P}}}{2}$ & $\begin{array}{ll}15.5 \quad(0.20) \\
\end{array}$ & $\begin{array}{ll}6.0 \quad(0.10) \\
\end{array}$ & $\begin{array}{ll}13.7 \quad(0.10) \\
\end{array}$ & $\begin{array}{ll}3.1 & (0.15) \\
\end{array}$ & $\begin{array}{ll}4.5 & (0.09) \\
\end{array}$ \\
\hline \multirow{6}{*}{$\mathrm{K}-\mathrm{NN}$} & $S_{0 \mathrm{~S}}$ & $\begin{array}{ll}20.2 \quad(0.22) \\
\end{array}$ & $\begin{array}{ll}9.1 \quad(0.09) \\
\end{array}$ & $\begin{array}{ll}9.7 & (0.09) \\
\end{array}$ & $\begin{array}{ll}22.9 \quad(0.25) \\
\end{array}$ & $\begin{array}{ll}7.8 \quad(0.12) \\
\end{array}$ \\
\hline & $s_{1 \mathrm{~S}}$ & $25.4 \quad(0.24)$ & $\begin{array}{ll}10.0 & (0.09)\end{array}$ & $15.9 \quad(0.11)$ & $\begin{array}{ll}6.6 & (0.14) \\
\end{array}$ & $\begin{array}{ll}7.2 & (0.12)\end{array}$ \\
\hline & $\frac{S_{0 \mathrm{~S}}+S_{1 \mathrm{~S}}}{2}$ & $\begin{array}{ll}15.3 \quad(0.20) \\
\end{array}$ & $\begin{array}{ll}7.4 \quad(0.09) \\
\end{array}$ & $11.1 \quad(0.09)$ & $\begin{array}{ll}14.2 \quad(0.21) \\
\end{array}$ & $\begin{array}{ll}3.3 \quad(0.10) \\
\end{array}$ \\
\hline & $S_{\mathrm{OP}}$ & $\begin{array}{ll}19.9 \quad(0.21) \\
\end{array}$ & $\begin{array}{ll}11.7 & (0.10) \\
\end{array}$ & $\begin{array}{ll}11.6 \quad(0.09) \\
\end{array}$ & $\begin{array}{ll}18.9 \quad(0.25) \\
\end{array}$ & $7.2 \quad(0.11)$ \\
\hline & $s_{1 \mathrm{P}}$ & $36.6 \quad(0.23)$ & $10.8 \quad(0.10)$ & $17.5 \quad(0.11)$ & $22.2 \quad(0.25)$ & $8.8 \quad(0.15)$ \\
\hline & $\frac{S_{0 \mathrm{P}}+S_{1 \mathrm{P}}}{2}$ & $18.1 \quad(0.21)$ & $10.4 \quad(0.10)$ & $12.9 \quad(0.10)$ & $\begin{array}{ll}8.8 & (0.19) \\
\end{array}$ & $\begin{array}{ll}6.8 \quad(0.12) \\
\end{array}$ \\
\hline SVM & Raw Data & $21.5 \quad(0.07)$ & $6.1 \quad(0.03)$ & $11.5 \quad(0.32)$ & $11.1 \quad(0.08)$ & $7.4 \quad(0.36)$ \\
\hline NFDA & Raw Data & $19.1 \quad(0.07)$ & $19.8 \quad(0.05)$ & $16.4 \quad(0.38)$ & $0.5 \quad(0.01)$ & $5.1 \quad(0.03)$ \\
\hline
\end{tabular}

distance in the raw data: $\sqrt{\sum_{i=1}^{T}\left(f_{a}(i)-f_{b}(i)\right)^{2}}$. Similarly, $S_{1 \mathrm{P}}$ denotes a pointwise similarity based on the Euclidean distance in the angle gived by the $n$ order tangent $\left(f^{n}\right): \sqrt{\sum_{i=1}^{T}\left(f_{a}^{n}(i)-f_{b}^{n}(i)\right)^{2}}$. Further, for the section-wise and point-wise similarities, linear combinations denoted by $\frac{S_{0 \mathrm{~S}}+S_{1 \mathrm{~S}}}{2}$ and $\frac{S_{0 \mathrm{P}}+S_{1 \mathrm{P}}}{2}$ were considered. Additionally to CABINTEC dataset, several well-known databases, out of the driving risk problem, were employed: the Gun Point, ECG200, Coffee, and Growth databases. A summary of these databases is shown in Table 2 (see [10 and 11 for a complete description). The linear segmentation algorithm proposed in Section 2 was applied to all the series.

In order to use Algorithm 1, the reduction parameter $\rho$ and the order of the tangent $n$ were established as 0.90 and 5 respectively in all cases. The parameter $R_{m i n}^{2}$ was selected by the minimization of (2) using train data. Two additional classifiers were considered and applied to the raw data: a SVM with a RBF kernel, and a classifier based in functional data representation (NFDA) [12]. Notice that k-NN applied to the point-wise similarity $S_{0 P}$, corresponds to the $\mathrm{k}-\mathrm{NN}$ evaluation on raw data. To compare the performance of the methods, the average classification results obtained in the test sets over 1000 runs were calculated. The mean error rate and its standard deviation in the test sets are presented in Table 3. In our case study, the CABINTEC database, the best global result was achieved by the combination of the section-wise similarities used as kernel for the SVM. The error reduction obtained by this combination regarding the individual similarities (10.2\% vs. $29.4 \%$ and $18.8 \%$ ) shows their complementarities in this database. Likewise, the combination of the point-wise 
similarities reached an error reduction. Similar results were obtained with the K-NN classifier. As a summary, in almost all the databases, the section-wise similarities or their combination achieved the best results. These results show the capacity of our method to reduce the complexity of the data, preserving the usefull information for classification purposes.

\section{Conclusions}

The main contribution of this paper, is a novelty methodology for the similarity measurement and classification of time series. A linear segmentation algorithm for the proper representation of subjective-data time series has been developed. Then, two similarity measures have been defined from the differences between the level of the series and the angle of the series, respectively. These similarities have been used as kernels to train a SVM. The application of the methodology to the CABINTEC database achieves outstanding results in the classification of traffic safety experts according to their experience. Moreover, very competitive results were achieved even in databases out of our study case. The results of the classification experiments show that the angle based similarities contain relevant information for classification purposes. This information, based on the behavior of the data, shows to be complementary to the information collected by the level based similarities. In this work, the section similarities were averaged over the total number of sections. In the future, the use of the individual similarities calculated in each section will be considered. In addition, the section-wise similarities could be used to detect clusters of experts when no label information is available.

Acknowledgments. Supported by the Minister for Science and Innovation of Spain: CABINTEC (PSE-37010-2007-2) and VULCANO (TEC2009-10639-C0404). Thanks to CONACYT and CONCYTEY for supporting the project through their scholarship programs.

\section{References}

1. Zhang, H., Schreiner, C., Zhang, K., Torkkola, K.: Naturalistic use of cell phones in driving and context-based user assistance. In: Proc. of the 9th Int. Conf. on Human Computer Interaction with Mobile Devices and Services, pp. 273-276 (2007)

2. Brazalez, A., et al.: CABINTEC: Cabina inteligente para el transporte por carretera. In: Proc. of the Congreso Español de Sistemas Inteligentes de Transporte (2008)

3. Tamil, E.M., et al.: A review on feature extraction \& classification techniques for biosignal processing (Part I: Electrocardiogram). In: Proc. of the 4th Kuala Lumpur Int. Conference on Biomedical Engineering 2008, vol. 21, pp. 117-121 (2008)

4. Keogh, E., Pazzani, M.: An enhanced representation of time series which allows fast and accurate classification, clustering and relevance feedback. In: KDD, pp. 239-243 (1998) 
5. Siordia, O.S., Martín, I., Conde, C., Reyes, G., Cabello, E.: Driving risk classification based on experts evaluation. In: Proceedings of the 2010 IEEE Intelligent Vehicles Symposium (IV 2010), pp. 1098-1103 (2010)

6. Randall, C., et al.: A comparison of the verbal rating scale and the visual analog scale for pain assessment. Technical Report 1, Int. Journal of Anesthesiology (2004)

7. Keogh, E., Chu, S., Hart, D., Pazzani, M.: Segmenting time series: A survey and novel approach. In: Data Mining in Time Series Databases, pp. 1-22 (1993)

8. Lachaud, J.-O., Vialard, A., de Vieilleville, F.: Analysis and comparative evaluation of discrete tangent estimators. In: Andrès, É., Damiand, G., Lienhardt, P. (eds.) DGCI 2005. LNCS, vol. 3429, pp. 240-251. Springer, Heidelberg (2005)

9. Martín, I., Muñoz, A., Moguerza, J.: Methods for the combination of kernel matrices within a support vector framework. Mach. Learn. 78, 137-174 (2010)

10. Keogh, E., Xi, X., Wei, L., Ratanamahatana, A.: The ucr time series classification/clustering (2006), http://www.cs.ucr.edu/ eamonn/time_series_data/

11. Ramsay, J., Silverman, B.: Functional Data Analysis. Springer Series in Statistics, Secaucus, NJ, USA (2005)

12. Ferraty, F., Vieu, P.: Nonparametric Functional Data Analysis: Theory and Practice. Springer Series in Statistics (2006) 